\title{
Hemothorax After Laparoscopic Cholecystectomy
}

\author{
René Scheer, MD, Pascal Steenvoorde, MD, PhD \\ Department of Surgery, Medisch Spectrum Twente, Enschede, The Netherlands (all authors).
}

\begin{abstract}
A patient who underwent a laparoscopic cholecystectomy presented 6 days postoperatively with chest pain on the right side. Further examination showed a hemothorax. This is a very rare complication of laparoscopic abdominal surgery. Video-assisted thoracoscopic surgery is efficient and safe in the treatment of this complication.
\end{abstract}

Key Words: Laparascopy, Cholecystectomy, Hemothorax, Complication, Video-assisted thoracoscopic surgery.

Citation Scheer R, Steenvoorde P. Hemothorax after laparoscopic cholecystectomy. CRSLS e2014.00049. DOI: 10.4293/CRSLS.2014.00049.

Copyright (C) 2014 SLS This is an open-access article distributed under the terms of the Creative Commons Attribution-Noncommercial-ShareAlike 3.0 Unported license, which permits unrestricted noncommercial use, distribution, and reproduction in any medium, provided the original author and source are credited.

Address correspondence to: René Scheer, MD, MST Department of Surgery, PO Box 50007500 KA Enschede, The Netherlands, E-mail: r.scheer@mst.nl

\section{INTRODUCTION}

In this case report we present a very rare complication of a laparoscopic cholecystectomy. The pathophysiologic principles and the treatment are discussed.

\section{CASE PRESENTATION}

A 43-year-old woman, with no relevant medical history besides severe pneumonia in childhood leading to hospital admission, was treated at our institution for symptomatic cholecystolithiasis. A laparoscopic cholecystectomy was performed. The subumbilical trocar was introduced bluntly, and pneumoperitoneum was kept constantly at the level of $12 \mathrm{~mm} \mathrm{Hg}$ during the procedure. The other ports were introduced under direct laparoscopic vision. There were no intraoperative complications, and intraoperative bleeding was nil. No impairment of respiratory parameters was observed by the anesthesiologist. The direct postoperative course was uncomplicated.

The patient presented to our emergency department 6 days after the procedure. She had chest pain on the right side with respiration. She was hemodynamically stable, and her oxygen level was 95\%. The percussion of the lower posterior chest was muted, and there were decreased breath sounds in this area. There were no signs of deep venous thrombosis.

A chest radiograph (Figure 1) and a supplementary thoracic and abdominal computed tomography scan showed a substantial amount of pleural effusion with atelectasis of the right lower lobe, an elevated right hemidiaphragm, no evidence of pulmonary embolism, and normal postoperative findings in the liver hilum.

A diagnostic pleural puncture was performed, whereby 650 $\mathrm{mL}$ of blood was drained at once. An in situ left thoracic drain produced $200 \mathrm{~mL}$ in the 2 hours thereafter. The hemoglobin concentration of the drained fluid was $7.2 \mathrm{mmol} / \mathrm{L}$ $(11.6 \mathrm{~g} / \mathrm{dL})$, confirming the diagnosis of hemothorax.

Despite drainage, the dyspnea persisted, as did the findings on chest radiographs. It was decided to perform video-assisted thoracoscopic surgery (VATS). No intraabdominal abnormalities were found on diagnostic laparoscopy through the diaphragm during this procedure.

Multiple blood clots were removed from the pleural cavity. There was persistent bleeding at the level of transition from the diaphragm to the pleural sinus. This bleeding was controlled by diathermic coagulation (Figure 2). Two chest drains were left behind, which could be removed 3 days after surgery. The postoperative course was uncomplicated. A control chest radiograph 2 weeks after surgery showed some residual defects of the right lung. A mild, asymptomatic restrictive lung function persisted at six months follow-up.

\section{DISCUSSION}

Thoracic bleeding as a complication after elective abdominal laparoscopic surgery is extremely rare. To our knowledge, only 2 cases have been described in the literature previously., ${ }^{1,2}$

Thoracic hemorrhage may be due to surgery directly, for example, as a result of unrecognized iatrogenic lesions of the diaphragmatic vasculature. On the other hand, it could be a 


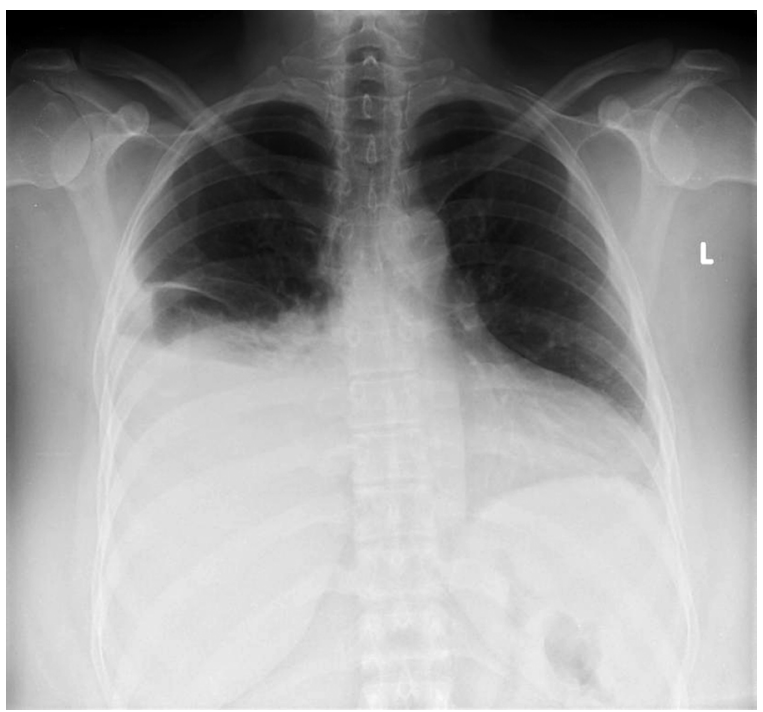

Figure 1. Chest radiograph at presentation in emergency department showing right-sided pleural effusion.

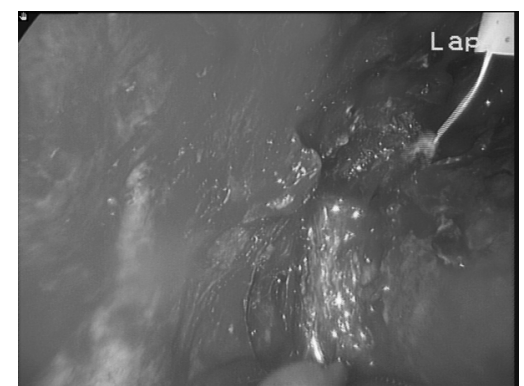

Figure 2. VATS with hemorrhagic focus at transition from diaphragm to pleural sinus.

complication of anesthesia. For example, a ventilation-related pneumothorax with rupture of vascular adherences between the parietal and visceral pleura could be a cause. However, these complications are expected to be seen perioperatively or to present with difficulties with ventilation or hemodynamic instability during or directly after the procedure.

The hemorrhage at the transition of the diaphragm to the right pleural sinus in our patient could be caused by a changed diaphragmatic position during the period of pneumoperitoneum, which has been seen on magnetic resonance imaging examination in pigs; however, in this case this is speculative. The cranial displacement of the diaphragm could have caused a laceration of the adherence of the parietal pleura with the diaphragm. ${ }^{3}$ A predisposing factor might be pneumonia, for which our patient was admitted to the hospital during childhood.

Our patient presented 6 days after the laparoscopic cholecystectomy. It is conceivable that there had been a precipitating moment, like coughing or physical activity, that caused laceration of a blood clot that covered the laceration of the vascular adherence at the first instance.

Attempting adequate drainage with a tube thoracostomy is the initial measure for a hemothorax. Persistent retained hemothorax should be treated with VATS between 3 and 7 days after hospitalization to decrease the risk of infection, development of a trapped lung with decreased lung function, or need for conversion thoracotomy. ${ }^{4}$

VATS is safe and effective in the treatment of hemothorax when surgery is necessary. 5 Patients should be hemodynamically stable. ${ }^{6}$ The procedure should be converted in the case of massive blood loss or if necessary to achieve an accurate surgical procedure. ${ }^{7-9}$

\section{References:}

1. Alcazar MT, Koo M, Ramiro O, Villalonga R, Sabate A, Montero A. Hemothorax as complication of laparoscopic technique. Rev Esp Anestesiol Reanim. 2003;50(3):150-153.

2. Cristian R, Massimiliano P, Tommaso R, et al. Massive right hemothorax as the source of hemorrhagic shock after laparoscopic cholecystectomy - case report of a rare intraoperative complication. Patient Saf Surg. 2011;5(1):12.

3. Sanchez-Margallo FM, Moyano-Cuevas JL, Latorre R, et al. Anatomical changes due to pneumoperitoneum analyzed by MRI: an experimental study in pigs. Surg Radiol Anat. 2011; 33(5):389-396.

4. Mowery NT, Gunter OL, Collier BR, et al. Practice management guidelines for management of hemothorax and occult pneumothorax. J Trauma. 2011;70(2):510-518.

5. Ambrogi MC, Lucchi M, Dini P, Mussi A, Angeletti CA. Videothoracoscopy for evaluation and treatment of hemothorax. J Cardiovasc Surg (Torino). 2002;43(1):109-112.

6. Manlulu AV, Lee TW, Thung KH, Wong R, Yim AP. Current indications and results of VATS in the evaluation and management of hemodynamically stable thoracic injuries. Eur J Cardiothorac Surg. 2004;25(6):1048-1053.

7. Lang-Lazdunski L, Chapuis O, Pons F, Jancovici R. Videothoracospy in thoracic trauma and penetrating injuries [in French]. Ann Chir. 2003;128(2):75-80.

8. DuBose J, Inaba K, Demetriades D, et al. Management of post-traumatic retained hemothorax: a prospective, observational, multicenter AAST study. I Trauma Acute Care Surg. 2012;72(1):11-22; discussion 22-24; quiz 316.

9. Heniford BT, Carrillo EH, Spain DA, Sosa JL, Fulton RL, Richardson JD. The role of thoracoscopy in the management of retained thoracic collections after trauma. Ann Thorac Surg. 1997;63(4):940-943. 